\title{
Does the Extent of Lymphadenectomy Have Impact on the Prognosis of Patients with Pancreatic Cancer?
}

\author{
D. Henne-Bruns ${ }^{\mathrm{a}} \quad$ I. Vogel ${ }^{\mathrm{b}}$ \\ aAbteilung für Viszeral- und Transplantationschirurgie, Universitätsklinik Ulm

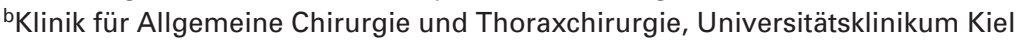

\section{Introduction}

Ductal adenocarcinoma is the most frequently occurring malignant tumor of the pancreas ( $80 \%$ of all cases) and has a most unfavorable prognosis. The incidence of pancreatic carcinoma in Western countries is about 10:100000 with a correlation to the patients' age (manifestation of $30 \%$ at the age of $50-60$ years and $25 \%$ at the age of $60-70$ years). In $2 / 3$ of patients the tumor is located in the pancreatic head and in $1 / 3$ in the corpus or tail [1]. According to the information of the Federal Statistical Office of Germany in 1997 pancreatic carcinoma is listed in rank 6 of statistics deaths caused by malignant diseases.

Because of the late onset of symptoms most pancreatic tumors are diagnosed in an advanced stage of the disease. The resection rates are therefore low, ranging worldwide between 10 and $20 \%$ [2]. Although nowadays resections can be performed with acceptable morbidity and mortality rates (less than 5\%) the overall 5-year survival rates after resection do not exceed more than $20 \%$ [3].

Since there are still no alternative curative treatment modalities, surgical resection of pancreatic carcinoma is the only therapeutic approach with a hope for cure. This resulted in the development of extensive surgical procedures over the last 20 years, but a positive influence of the extended radicalness on survival rates is still being discussed controversially.

\section{Surgical Technique}

\section{Extent of Lymphadenectomy}

The curative surgical therapy of pancreatic carcinoma includes resection of the involved part of the pancreas and the locoregional lymphatic tissues.
Tumors of the pancreatic head are treated with partial duodenopancreatectomy, tumors of the corpus/tail region with a left-sided pancreatic resection and splenectomy. Because of consequently arising problems in connection with complete exo- and endocrinological insufficiency, a total pancreatectomy is only indicated when the entire pancreas is involved.

Since isolated resection of the pancreatic tumor could not improve long-term survival, over the last 15 years surgical procedures were extended. Operations including intra- and retroperitoneal lymphadenectomies were performed. This extension of the surgical procedure is (among others) based on the findings of Ishikawa et al. [4], who in 1988 described that patients with a resectable pancreatic head carcinoma showed an improvement in the 5-year survival time from 9 to $28 \%$ after radical intra- and retroperitoneal lymphadenectomy.

For our own operative strategy we distinguish between regional and extended retroperitoneal lymphadenectomy. Regional lymphadenectomy includes resection of the pancreatic head with removal of all lymphatic tissue at the hepatoduodenal ligament, the hepatic artery, the celiac trunk, the first $3 \mathrm{~cm}$ of the splenic artery, the right side of the superior mesenteric artery, and the ventral surface of the vena cava and the renal veins.

During extended retroperitoneal lymphadenectomy (as described by Ishikawa et al. [4]) all lymphatic, connective and neural tissues at the aorta between the inferior mesenteric artery and the coeliac trunk as well as the left side of the superior mesenteric artery are additionally resected.

Recently published studies [5] as well as our own data [6] do not allow a definitive conclusion regarding the prognostic impact of extended retroperitoneal resection on the prognosis of the patients. The analysis of the prognostic factors for longterm survival in our own 72 patients with ductal adenocarcinoma of the pancreatic head operated between 1988 and 1998 (regional LA $n=26$, extended LA $n=46$ ) could not demon-

\begin{tabular}{ll}
\hline KARGER & @ 2002 S. Karger GmbH, Freiburg \\
Fax +49 7614520714 & Accessible online at: \\
$\begin{array}{l}\text { E-mail Information@Karger.de } \\
\text { www.karger.com }\end{array}$ & www.karger.com/journals/onk
\end{tabular}

Prof. Dr. med. Doris Henne-Bruns

Abteilung für Viszeral- und Transplantationschirurgie, Universitätsklinik Ulm Steinhövelstraße 9, D-89070 Ulm (Germany)

Tel. +49 731500 272-00, Fax -09

E-mail doris.henne-bruns@medizin.uni-ulm.de 
strate a prognostic impact of extended lymphadenectomy on survival, but for patients with early tumor stages (UICC-stage I/II, $n=18$ ) a 5 -year survival of $63 \%$ could be reached. In this group of patients no difference between regional and extended retroperitoneal lymphadenectomy could be observed, so it must be concluded that regional lymphadenectomy positively influences the course of disease in the early stage [6].

A direct comparison with the results of other authors is difficult because the following parameters vary considerably in the different studies:

- The operative procedures (extent of lymphadenectomy, principally performed resection of the portal vein).

- The histopathological analysis and diagnosis (preparation of the resected tissue, number of analyzed lymph nodes, exact localization of the lymphatic tissue, differentiation of the tumor).

- The percentage of ductal adenocarcinoma of the pancreas in the analyzed group of patients.

- The UICC-tumor stages of the patients with ductal adenocarcinoma of the pancreas in the studies.

- The percentage of R0-resected patients.

On the other hand it can not be excluded that the improvement of the 5-year survival rates after extended retroperitoneal lymphadenectomy is based on the phenomenon of 'stage migration'. The analysis of a larger number of resected lymph nodes results most likely in a more precise (mostly higher) tumor staging with the following effect: There are no longer patients with a lymph node invasion and thus a more negative prognosis in the stages of patients without invasion. Consequently, the survival rate of this group is improved. Furthermore, there will be an increase of patients with just one positive lymph node (N1a-stage) in the group of patients with positive lymph nodes, thus improving the 5-year survival rate in this group also. Altogether, extended lymphadenectomy seems to lead to an improved prognosis. In reality, however, only a more exact staging exists.

The answer to the question whether and to what extent lymphadenectomy influences the prognosis for patients with ductal adenocarcinoma of the pancreas is principally very important, since radical lymphatic dissection, as described by Ishikawa et al. [4] may lead to considerable postoperative disorders. These can be motility disturbances and, in some cases, long-term diarrhea necessitating hospitalization.

Further study protocols will have to face all these questions in a multicenter study in regard to criteria for surgery, extent of lymphadenectomy and histopathology.

In 1998 the International Workshop on Surgical Procedures in Pancreatic Cancer tried to define standards of terminology for the operative procedures and histopathological evaluation [7]. For the surgical approach concerning the extent of lymphadenectomy three types were defined: standard procedure, radical lymphadenectomy and extended radical lymphadenectomy. The extent of the resected lymph nodes was defined according to the Japanese classification, because only there dif- ferent stations are described in detail. On this basis a multicenter study was initiated, the results of which are still to be expected.

\section{Resection of the Portal Vein}

Another open question is the indication for pancreatic head resection in patients with preoperatively diagnosed portal invasion. From the surgeon's point of view the segmental resection of the portal vein / vena mesenterica superior and their reconstruction do not substantially extend the operation and increase perioperative morbidity and mortality, but the value of the resection regarding long-term survival can not be evaluated so far.

Ishikawa et al. [8] described a 3-year survival rate of 59\% in patients with a short infiltration of the portal vein. This is in contrast to other authors and our own experience [6] where even after R0 resection none of the patients with portal infiltration survived longer than 12 months.

The remaining problem is the selection criteria for a curative resection, since imaging procedures might demonstrate a stenosis of the portal vein, but mostly are not able to histologically differ between compression and infiltration. As our own results demonstrate, even intraoperatively, during preparation or even resection, a definitive judgement can be difficult. In 20 of the 72 patients of our group the portal vein was resected and reconstructed, but in only 16 cases an infiltration was proven, while in 4 cases only an inflammation could be found in the histopathological examination.

Because of the difficulty to evaluate a surface wall infiltration and the potential risk of microscopically small residual tumor masses, some Japanese work groups consequently resect the portal vein in every pancreatic resection. This procedure was also described by American authors, namely Fortner [9].

\section{Disseminated Tumor Cells}

Quite a number of work groups recently demonstrated that micrometastases and single disseminated tumor cells can be detected by immunocytological and molecularbiological methods, which has not been possible by conventional histological techniques. Single tumor cells can be detected in lymph nodes, bone marrow, blood, peritoneal cavity and in secretory products (e.g. by the gall bladder). The biological relevance of these single cells is not yet clear. Can they proliferate and mutate to metastases? Are they in a resting position with the possibility to proliferate any time? These questions are to be answered.

Vogel and Kalthoff [1] could clearly demonstrate in a survey of more than 80 patients with pancreatic carcinoma that a correlation between detection of single tumor cells and tumor stage exists and that the 5-year survival rate is significantly better for patients without verified micrometastases. 
Thus, tumor cell dissemination seems to have a prognostic relevance which has not yet been taken into consideration in the present TNM classification. This will, however, reach great importance for the exact tumor staging and $\mathrm{R}$ classification in the future.

The extent of the surgical intervention regarding radical lymphadenectomy will definitely have to be discussed after data on the prognosis factor 'disseminated single tumor cell' are available.

\section{Conclusion}

Tumor resection is presently the only curative treatment option in pancreatic carcinoma.

In some published studies the development of extended surgical procedures (radical lymphadenectomy) resulted in prolonged survival. The effect of the phenomenon 'stage migration' remains to be clarified. Meanwhile, the value and extent of the lymphadenectomy necessary for prognosis improvement can not yet be determined.

Our own concept includes a regional lymphadenectomy in all cases. This procedure includes removal of the lymphatic nodes at the hepatoduodenal ligament, the celiac trunk, the first part of the A. lienalis, the right side of the A. mesenterica superior, and the ventral part of the $\mathrm{V}$. cava under preparation of the renal vein junction. The reason for this procedure is an exact staging of the lymphatic nodes, which we send to the patholo- gist separately. This procedure also minimized the risk of severe postoperative diarrhea because it preserves the left lymphatic tissue of the superior mesenteric artery.

Extended lymphadenectomy with additional resection of the paraaortal lymphatic tissue and the lymph nodes on the left side of the mesenteric artery results in higher postoperative morbidity due to mobility disturbances and diarrhea. An increase in long-term survival due to a higher radicalness so far has not been proven, the risk of reduced quality of life is clearly increased.

Therefore extended resection should only be performed in studies after informing the patient in detail and evaluating the postoperative quality of life.

Even if there remain open questions concerning the extent of surgical intervention, the basic principle of surgical intervention in curative intention persists. $\mathrm{R} 0$ resection of the tumor (marcroscopically and microscopically no tumor residua) is essential for the chance of long term survival and depends on early tumor stages. R1 and R2 resections clearly have a negative prognosis. Therefore, an extension of the surgical procedure including gastrectomy, colonic resection or resection and reconstruction of major vessels is justified if a total removal of the primary tumor can be achieved.

With advanced tumor stages (and in patients with proven tumor cell dissemination?!) resection alone will not lead to improvement of survival and therefore has to be combined with effective adjuvant therapies (chemo-, radio-, immunotherapy) to increase the survival chance of these patients.

\section{References}

1 Vogel I, Kalthoff H: Disseminated tumor cells: Their detection and significance for prognosis of gastrointestinal and pancreatic carcinomas. Virchows Arch 2001;439:109-117.

2 Bramhall SR, Allum WH, Jones AG, Allwood A, Cummins C, Neoptolemos JP: Treatment and survival in 13,560 patients with pancreatic cancer, and incidence of the disease, in the West Midlands: An epidemiological study. Br J Surg 1995;82:111-115.

3 Warschaw AL, Fernández-Del Castillo C: Pancreatic carcinoma. N Engl J Med 1992;326:455-465.
4 Ishikawa O, Ohigashi H, Sasaki Y, Kabuto T, Fukuda I, Furukawa H, Imaoka S, Iwanaga T: Practical usefulness of lymphatic and connective tissue clearance for the carcinoma of the pancreatic head. Ann Surg 1988;208:215-220.

5 Birk D, Beger HG: Lymph-node dissection in pancreatic cancer - what are the facts? Langenbecks Arch Surg 1999;384:58-166.

6 Henne-Bruns D, Vogel I, Lüttges J, Klöppel G, Kremer B: Surgery for ductal adenocarcinoma of the pancreatic head: Staging, complications and survival after regional versus extended lymphadenectomy. World J Surg 2000;24:595-602.
7 Pedrazzoli S, Beger HG, Obertop H, Andren-Sandberg A, Fernandez-Del Casillo C, Henne-Bruns D, Lüttges J, Neoptolemos JP: A surgical and pathological based classification of resective treatment of pancreatic cancer. Dig Surg 1999;16:337-345.

8 Ishikawa O, Ohigashi H, Imaoka S, Furukawa H, Sasaki Y, Fujita M, Kuroda C, Iwanaga T: Preoperative indications for extended pancreatectomy for locally advanced pancreas cancer involving the portal vein. Ann Surg 1992;215:231-236.

9 Fortner JG: Regional pancreatectomy for cancer of the pancreas, ampulla, and other related sites. Ann Surg 1984;199:418-425. 